\title{
MORITA EQUIVALENCE AND INFINITE MATRIX RINGS
}

\author{
VICTOR CAMILLO
}

\begin{abstract}
This paper contains the proof of a theorem conjectured by William Stephenson in his thesis [3].
\end{abstract}

Two rings $R$ and $S$ are Morita equivalent if their categories of right $R$ modules are isomorphic. A standard example of two Morita equivalent rings are $R$ and $R_{n}$, the $n \times n$ matrices over $R$. It is therefore true that if $R_{n}$ and $S_{m}$ are isomorphic for integers $n$ and $m$ then $R$ and $S$ are Morita equivalent. An easy example (below) shows that there are Morita equivalent rings $R$ and $S$ with $R_{n}$ and $S_{m}$ not isomorphic for any integers $m$ and $n$. This means that category isomorphism cannot be reduced to a ring isomorphism in this way. Regard $R_{n}$ as End $R^{(n)}$, where $R^{(n)}$ is a direct sum of $n$ copies of $R$. Let $R^{(N)}$ be a direct sum of countably many copies of $R$. It follows from a result of Eilenberg (below) that if $R$ and $S$ are Morita equivalent, then End $R^{(N)} \approx$ End $S^{(N)}$. We prove the following

TheOrem. $R$ and $S$ are Morita equivalent if and only if End $R^{(N)} \approx$ End $S^{(N)}$, where $N$ is a countably infinite set.

Note End $R^{(N)}=R_{N}$ is just the ring of column finite matrices over $R$.

The following example was suggested by Kent Fuller. I would also like to thank him for several helpful conversations.

EXAMPle. Two Morita equivalent rings $R$ and $S$ with $R_{n}$ not isomorphic to $S_{m}$ for any integers $n$ and $m$.

Let $K$ be any field, $R \approx K \times K$ and $S \approx K \times K_{2}$. Then $R_{n} \approx K_{n} \times K_{n}$ and $S_{m} \approx K_{m} \times K_{2 m}$. By the uniqueness part of the Wedderburn theorem, the latter two rings cannot be isomorphic, but $R$ and $S$ are clearly Morita equivalent.

Our result is motivated by, and requires, a result of Eilenberg. This is an exercise in Anderson and Fuller [1, p. 202]. Let $R$ be a ring. Recall that $P$ is a progenerator if $P$ is a finitely generated projective generator, that is, $R^{(n)} \approx P \oplus K$ and $P^{(m)} \approx R \oplus L$ for modules $K$ and $L$, and integers $m$ and $n$.

NotAtion. $X^{(N)}$ always denotes the direct sum of a countable number of copies of $X$.

For completeness we sketch a proof of the following

Proposition (EIlenberg). If $P$ is a progenerator, $P^{(N)} \approx R^{(N)}$.

We use the notation preceding the proposition, and note that without loss of generality $n=m$. Only one substitution is made on each line.

Received by the editors January 25, 1983 and, in revised form, June 23, 1983.

1980 Mathematics Subject Classification. Primary 16A89, 16A49; Secondary 16A36, 16A42.

(C)1984 American Mathematical Society $0002-9939 / 84 \$ 1.00+\$ .25$ per page 
We have

$$
P^{(N)} \approx R^{(N)} \oplus L^{(N)} \approx R^{(N)} \oplus R^{(N)} \oplus L^{(N)} \approx R^{(N)} \oplus P^{(N)}
$$

Similarly, $R^{(N)} \approx P^{(N)} \oplus R^{(N)}$, and we are done.

To obtain our results we will need an observation about projective modules. If $X$ is any module and $\left\{Y_{i}\right\}$ is an infinite set of modules, then $G=\operatorname{Hom}\left(X, \Sigma \oplus Y_{i}\right)$ may clearly be described as the subgroup of $\Pi \operatorname{Hom}\left(X, Y_{i}\right)$ with the property that $f=\left(f_{1}, \ldots, f_{n}, \ldots\right)$ is in $G$ if and only if for every $x \in X, f_{i}(x)=0$ for all but finitely many $i$. We thus have a canonical embedding

$$
c: \sum \oplus \operatorname{Hom}\left(X, Y_{i}\right) \rightarrow \operatorname{Hom}\left(X, \sum \oplus Y_{i}\right) \text {. }
$$

If $X$ is finitely generated then $c$ is obviously an isomorphism. For projective modules we observe:

Lemma 1. Let $P_{R}$ be a projective module. Then $P_{R}$ is finitely generated if and only if the $c$ obtained from the map above by letting $X=P$ and $Y_{i}=P$ is an isomorphism.

Proof. Recall that $P$ is projective if and only if $P$ has a dual basis, i.e., a set $\left\{\left(p_{i}, f_{i}\right)\right\}$ where $p_{i} \in P$ and $f_{i} \in \operatorname{Hom}(P, R)$, such that for a given $p \in P$ :

1. $f_{i}(p)=0$ for almost all $i$ and

2. $p=\sum p_{i} f_{i}(p)$.

Now if $c$ is an isomorphism, the map $g: P \rightarrow P^{(N)}$ given by $g(p)=$ $\left(p_{1} f_{1}(p), \ldots, p_{n} f_{n}(p), \ldots\right)$ must be identically zero from some point on, so clearly $P$ is finitely generated. As observed in the remark preceding the lemma, the converse is obvious.

Before we prove our theorem we recall the Morita theorem.

Morita TheOrem. Let $R$ and $S$ be rings. Then $R$ and $S$ have isomorphic categories of right modules if and only if there is a finitely generated projective generator $P_{R}$ such that $S=$ End $P_{R}$. The category isomorphism is given by $X_{R} \rightarrow \operatorname{Hom}\left(P_{R}, X_{R}\right)$.

The functor $\operatorname{Hom}\left(P_{R},-\right)$ does the usual on maps.

Before we prove our theorem, let us take a look at Eilenberg's proposition. If we take the endomorphism ring of both sides of the isomorphism in the proposition, we obtain $R_{N} \approx S_{N}$, where $S=$ End $P$. Note, End $X^{(N)} \approx(\text { End } X)_{N}$ in general only when $X$ is finitely generated. Thus, by Morita's theorem, if $R$ and $S$ are Morita equivalent, $R_{N} \approx S_{N}$. This was the motivation for our theorem. What we need to do is, given an isomorphism between the column finite matrices, pick out a progenerator that works.

Proof of The Theorem. Notation: $U=R^{(N)}=\Sigma \oplus u_{i} R ; V=S^{(N)}=\Sigma \oplus v_{i} S$; $\left\{e_{i j}\right\}$ are the usual matrix units for $U$, i.e., $e_{i j}\left(u_{j}\right)=u_{i}$ and $e_{i j}\left(u_{k}\right)=0$ if $k \neq j .\left\{f_{i j}\right\}$ are the corresponding matrix units for $V$. Note that in this case, $\sum e_{i i}$ and $\sum f_{i i}$ makes not sense. Finally, $\sigma$ is an isomorphism from $R_{N}$ to $S_{N}$.

To establish our result we need to find and $S$ progenerator $P$ with $R=$ End $P$. There is an obvious candidate; $\sigma\left(e_{11}\right)$ is an idempotent in End $V$, therefore $V=$ $\sigma\left(e_{11}\right) V \oplus C$, where $C$ is a complement. $\sigma\left(e_{11}\right) V$, being a summand of a free module, is projective, but not obviously finitely generated nor a generator. We prove this. 
$\sigma\left(e_{11}\right) V$ is finitely generated. We first note that $\sigma\left(e_{i i}\right) V \approx \sigma\left(e_{11}\right) V$ for all $i$. This is just a standard calculation with matrix units. Let $h_{i j}=\sigma\left(f_{i j}\right)$;

$$
h_{i i} V \supset h_{i i} h_{i j} V=h_{i j} V \supset h_{i j} h_{j i} V=h_{i i} V \text {, }
$$

so $h_{i i} V=h_{i j} V$. So, $h_{j i}\left(h_{i i} V\right) \subset h_{j j} V$ and $h_{i j}\left(h_{j j} V\right) \supset h_{i i} V$, and the composition of the two maps given by $e_{j i}$ and $e_{i j}$ is the identity on $e_{i i} V$.

We therefore have that $\sigma\left(e_{11}\right) V \approx \sigma\left(e_{i i}\right) V$ for all $i$, and note that $\sum \sigma\left(e_{i i}\right) V$ is direct. Now, if $\sigma\left(e_{11}\right) V$ is not finitely generated, we may construct a map $h: \sigma\left(e_{11}\right) V \rightarrow$ $\sum \sigma\left(e_{i i} V\right)$ as in the lemma, using a dual basis for $\sigma\left(e_{11}\right) V$. $h$ has the property that $\operatorname{Im} h$ is not contained in any finite sum of the $\sigma\left(e_{i i}\right) V$. Now, since $\sigma\left(e_{11}\right) V$ is a summand for $V$ we can extend the domain of $h$ to all of $V$ by making it zero on the complement, which we still call $h$.

Now, go back to $U$, and look at $\sigma^{-1}(h) e_{11}$. We know that $\sigma^{-1}(h) e_{11} u_{i}=0$ for $i \neq 1$, so $\sigma^{-1}(h) e_{11} U=\left(\sigma^{-1}(h) e_{11} u_{1}\right) R$, and this latter module is principal, therefore it is contained in a finite sum $\sum_{i=1}^{K} u_{i} R$. Therefore, $e_{11} \sigma^{-1} h e_{11}=0$ for all $i>K$, so $\sigma\left(e_{i i}\right) \sigma\left(\sigma^{-1}(h)\right) \sigma\left(e_{11}\right)=0$ for all $i>k$, i.e., $\sigma\left(e_{i i}\right) h \sigma\left(e_{11}\right)=0$ for all $i>K$, a contradiction, which shows that $\sigma\left(e_{11}\right) V$ is finitely generated.

$\sigma\left(e_{11}\right) V$ is a generator. Look at $\sigma^{-1}\left(f_{11}\right)$. By the previous argument, $\sigma^{-1}\left(f_{11}\right) U$ is finitely generated, and so is contained in a finite sum of the $u_{i} R$. Thus there is a $K$ with

$$
\left(e_{11}+\cdots+e_{K K}\right) \sigma^{-1}\left(f_{11}\right)=\sigma^{-1}\left(f_{11}\right) .
$$

So, applying $\sigma$, we have $\left(\sigma\left(e_{11}\right)+\cdots+\sigma\left(e_{K K}\right)\right) f_{11}=f_{11}$. Multiply on the left to get

$$
\left[f_{11} \sigma\left(e_{11}\right)+\cdots+f_{11} \sigma\left(e_{K K}\right)\right] f_{11}=f_{11} \text {. }
$$

Now evaluate at $v_{1}$ to get

$$
\left[f_{11} \sigma\left(e_{11}\right)+\cdots+f_{11} \sigma\left(e_{K K}\right)\right] v_{1}=v_{1} .
$$

Now let $G=\sigma\left(e_{11}\right) V \oplus \cdots \oplus \sigma\left(e_{K K}\right) V$ (external direct sum), and consider the map: $f: G \rightarrow v_{1} S$ given by

$$
\left(\sigma\left(e_{11}\right) v_{1}, \ldots, \sigma\left(e_{K K}\right) v_{K}\right) \rightarrow f_{11}\left(\sigma\left(e_{11}\right) v_{1}+\cdots+\sigma\left(e_{K K}\right) v_{K}\right) .
$$

Then (1) shows that $f$ is an epimorphism. Since $v_{1} S \approx S, f$ splits, and since $\sigma\left(e_{i i}\right) V \approx \sigma\left(e_{11}\right) V$, we have $\left[\sigma\left(e_{11}\right) V\right]^{(K)} \approx S \oplus C$, so $\sigma\left(e_{11}\right) V$ is a generator.

End $\sigma\left(e_{11}\right) V \approx R$. Let $M$ be any module and $M=N \oplus C$. Let $e$ be the projection onto $M$ along $C$. Then it is well known and easy to prove that End $N \approx e($ End $M) e$, so since $V=\sigma\left(e_{11}\right)(V) \oplus C$, End $\sigma\left(e_{11}\right) V=\sigma\left(e_{11}\right)($ End $V) \sigma\left(e_{11}\right)$. Apply $\sigma^{-1}$ :

$$
\text { End } \sigma\left(e_{11}\right) V \approx e_{11} \sigma^{-1}(\text { End } V) e_{11}=e_{11} R_{N} e_{11} \approx R \text {. }
$$

\section{REFERENCES}

1. F. Anderson and K. Fuller, Rings and categories of modules, Springer-Verlag, New York, 1973.

2. K. Morita, Duality of modules and its applications to the theory of rings with minimum condition, Sci. Rep. Tokyo Kyoiku Daigaku Sect. A 6 (1958), 85-142.

3. W. Stephenson, Ph. D. Thesis, Univ. of London, 1967.

Department of Mathematics, University of Iowa, Iowa City, Iowa 52242 